\title{
NONLINEAR FAR-INFRARED PHOTOIONIZATION OF SHALLOW ACCEPTORS IN GERMANIUM
}

\author{
G. Jungwirt and W. Prettl \\ Instıtut für Angewandte Physik der Universität Regensburg, D-8400 Regensburg, Deutschland
}

\begin{abstract}
The optical saturation of nonresonant photoionization of shallow acceptors in $p$-Ge was investigated at low temperatures using a high-power FIR molecular laser. The experimental results were analyzed in terms of a rate equation model. Very small saturation intensities in the order of $10 \mathrm{~mW} / \mathrm{cm}^{-2}$ and long free hole recombination times in the range of $10 \mu \mathrm{s}$ were observed.
\end{abstract}

\section{INTRODUCTION}

Nonlinear dynamical properties of carrier transport like self-sustained oscillations and chaotic fluctuations in semiconductors are of strong current interest. ${ }^{(1)}$ In order to understand dynamical phenomena in high-purity semiconductors on a microscopic basis, detailed knowledge of the kinetics of charge carriers is needed. Free carrier relaxation times have been studied by various methods like the decay of conductivity after short voltage ${ }^{(2)}$ or laser ${ }^{(3,4)}$ pulses, generation-recombination noise ${ }^{(5)}$ and the band width of high frequency modulated far-infrared (FIR) photoconductivity. ${ }^{(6)}$ A powerful means to study the kinetics of carriers bound to shallow impurities represents saturation spectroscopy applying high-power FIR-lasers at low temperatures. Extensive investigations have been performed on $\mathrm{n}-\mathrm{GaAs}$ epitaxial layers. As a function of laser intensity incident on the sample, the absorption coefficient $t^{(7.8)}$ and the photoconductive signal ${ }^{(9.4)}$ due to resonant shallow donor transitions, nonresonant photoionization of shallow donors and cyclotron resonance ${ }^{(10)}$ were measured for various magnetic field strengths. The experimental results on optical transitions to impurity bound states were analyzed by appropriate rate equation models based on the photothermal free carrier generation process. ${ }^{(11)}$ At sufficiently low temperatures where thermal ionization of excited impurity states is strongly reduced, excited state impact ionization has been shown to be the dominant mechanism leading to a photoconductive signal. ${ }^{(12)}$

Basically optical saturation of shallow impurity transitions at low temperatures is determined by the recombination time i.e. the average time an excited carrrier needs to return to the ground state. This time must be distinguished from the decay time of conductivity due to short electric or laser pulses. The latter time constant is the life time of carriers in the band and corresponds to the average time free carriers are captured in high excited states. Both time constants may be very different.

Here we report on the optical saturation of photoionization of shallow acceptors in very pure and low compensated germanium. Previous investigations of optical transitions to metastable acceptor states, which are created by a magnetic field inside the valence band below each Landau level, ${ }^{(13)}$ showed that in the same material the optically generated concentration of free holes may exceed the density of compensating donors for very low intensities in the order of a few $\mathrm{mW} / \mathrm{cm}^{2}$. This leads to bimolecular free carrier recombination yielding a square root dependence of the photoconductive signal on intensity. ${ }^{(14)}$ In the present case of optical transitions from the donor ground state to continuum states of the valence band at zero magnetic field, a simple saturation behaviour like that of a two level system is observed indicating linear recombination of free carriers. Due to the small density of compensating donors, extremely small saturation intensities with correspondingly long free hole recombination times are observed.

\section{EXPERIMENTAL}

The measurements were carried out with a high-purity p-Ge sample containing predominantly the shallow acceptor boron. The effective acceptor concentration and the compensation ratio were 
$N_{\mathrm{A}}-N_{\mathrm{D}}=2.5 \cdot 10^{12} \mathrm{~cm}^{-3}$ and $N_{\mathrm{D}} / N_{\mathrm{A}}=0.01$, respectively. Ohmic contacts were prepared by alloying $\mathrm{Al}$ on opposite edges. The sample was immersed in liquid helium at $4.2 \mathrm{~K}$ in an optical cryostat. An electrically pulsed low pressure $\mathrm{CO}_{2}$ laser was used to pump a $\mathrm{CH}_{3} \mathrm{OH}$ FIR laser. Experimental results are presented for $\lambda=70 \mu \mathrm{m}$. Long laser pulses of $150 \mu \mathrm{s}$ duration were applied in order to have steady state conditions during irradiation. The intensity of the laser beam in the sample was determined by a calibrated pyroelectric detector taking into account the losses in the cryostat windows and the reflection at the sample surface. The intensity could be varied by calibrated absorbers. In any case the absorption length of the sample was much smaller than the thickness, thus variations of the intensity inside the sample may be neglected. The photoconduclive signal was measured with a standard load resistor circuit and recorded by a boxcar. The load resistor was much smaller than the sample resistance therefore the relative change of the bias voltage $V$ across the sample, $\Delta V / V$, is proportional to the change of the sample conductance. Assuming that the mobility varies only little, it follows that $\Delta V / V$ is proportional to the optically generated free hole concentration $\Delta p$. In the whole range of intensity the photoconductance was substantially larger than the dark conductance of the sample at $4.2 \mathrm{~K}$, therefore $\Delta p$ may be identified with the total concentration of free holes.

The photoconductivity spectrum measured by a Fourier transform spectrometer at $4.2 \mathrm{~K}$ is displayed in Fig. 1. The spectral lines are due to photothermal ionization caused by transitions from the acceptor ground state to excited bound states below the valence band edge. Additionally the photoionization threshold equal to the binding energy of boron $E_{\mathrm{l}}=87 \mathrm{~cm}^{-1}$, and the continuum spectrum $h \omega>E_{1}$ are clearly observed. The frequency corresponding to the wavelength of the applied laser line $\lambda=70 \mu \mathrm{m}$ is indicated by an arrow. In Fig. 2 the photoconductive signal is plotted as function of intensity obtained at temperatures $4.2 \mathrm{~K}$ [Fig. 2(a)] and $2 \mathrm{~K}$ [Fig. 2(b)]. In both cases measurements are shown for two different bias voltages being well below the impact ionization instability ( $V=3 \mathrm{~V}$ at $4.2 \mathrm{~K}$ ). The relative photoconductive signal $\Delta V / V$ is larger for higher bias voltage in contrast to a linear photoconductor. The nonlinear dependence of the signal on intensity I can be described by the simple relation $\Delta V / V \approx I \cdot\left(1+I / I_{\mathrm{S}}\right)^{-1}$ in excellent agreement to the measurements. The full lines in Fig. 2 show fits yielding extremely small saturation intensities summarized in Table 1. Compared e.g. to the nonresonant photoionization of shallow donors in

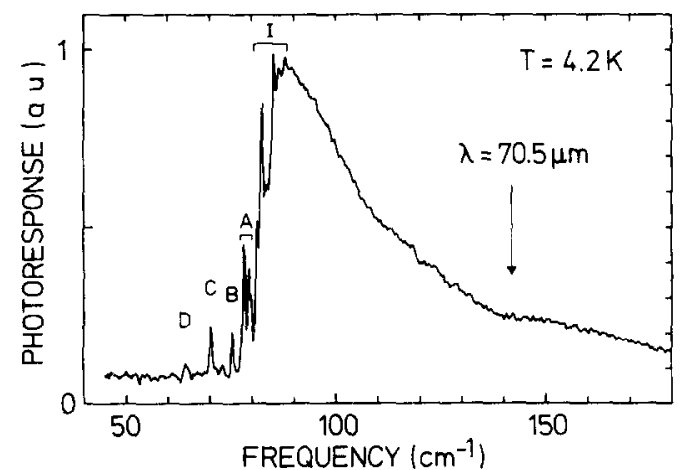

Fig. 1. Photoconductivity spectrum of p-Ge at 4.2 K. The notation of transitions to bound states is after Jones et al. ${ }^{15}$ The frequency corresponding to $\lambda=70 \mu \mathrm{m}$ is indicated by an arrow.

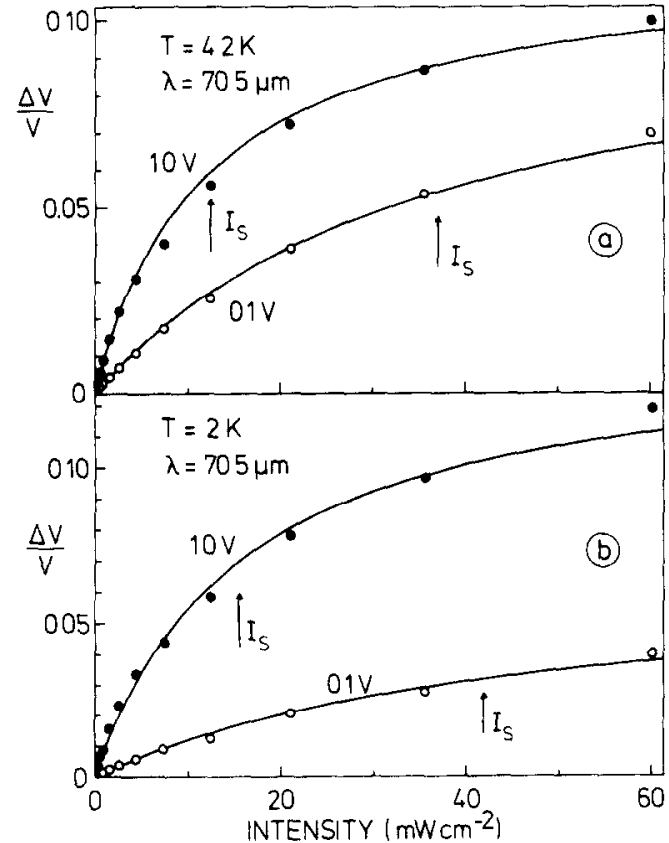

Fig. 2. Relative photoconductive signal $\Delta V / V$ as function of intensity for two bras voltage at (a) $42 \mathrm{~K}$ and (b) $2 \mathrm{~K}$. Solid and open circles are measured values and full lines are calculated. Saturation intensities are indicated by arrows 
Table 1. Saturation intensities and recombination times

\begin{tabular}{lcccc}
\hline Temperature & \multicolumn{2}{c}{$4.2 \mathrm{~K}$} & $\mathrm{~K}$ \\
\hline Bias voltage & $0.1 \mathrm{~V}$ & $1.0 \mathrm{~V}$ & $0.1 \mathrm{~V}$ & $1.0 \mathrm{~V}$ \\
Saturation intensity & $37 \mathrm{~mW} / \mathrm{cm}^{2}$ & $12 \mathrm{~mW} / \mathrm{cm}^{2}$ & $45 \mathrm{~mW} / \mathrm{cm}^{2}$ & $16 \mathrm{~mW} / \mathrm{cm}^{2}$ \\
Recombination time & $11 \mu \mathrm{s}$ & $34 \mu \mathrm{s}$ & $9 \mu \mathrm{s}$ & $26 \mu \mathrm{s}$ \\
\hline
\end{tabular}

$\mathrm{n}$-GaAs epitaxial layers, the saturation in the present material occurs at intensities more than one order of magnitude smaller. ${ }^{(4)}$

\section{THEORY AND DISCUSSION}

The experimental results are analyzed in terms of a generation-recombination model. Free holes are excited into the valence band by the transition probability $\sigma F$ where $\sigma$ is the photoionization cross section and $F=I / \hbar \omega$ is the photon flux density. Thermal generation of free carriers may be neglected because the temperature is much smaller than the binding energy of acceptors. According to the giant trap theory, ${ }^{(16)}$ free carriers are captured by high excited impurity states and return to the ground state across intermediate energy levels by emission of phonons. This cascade recombination of holes is modelled by introducing one representative excited state of sticking probability one which captures free holes with the rate $T_{1}^{\mathrm{S}} p n_{\mathrm{A}}$ where $p$ is the free hole concentration and $n_{\mathrm{A}}$ is the density of ionized acceptors. The excited state population $p_{\mathrm{A}}^{*}$ relaxes to the ground state with the rate $T_{2}^{\mathrm{S}} p_{\mathrm{A}}^{*}$. The coefficients $T_{1}^{\mathrm{S}}$ and $T_{2}^{\mathrm{S}}$ generally depend on temperature and bias voltage. The rate equations according to these considerations are

$$
\begin{aligned}
\frac{\mathrm{d} p}{\mathrm{~d} t} & =\sigma F p_{\mathrm{A}}-p T_{1}^{\mathrm{S}} n_{\mathrm{A}} \\
\frac{\mathrm{d} p_{\mathrm{A}}^{*}}{\mathrm{~d} t} & =p T_{1}^{\mathrm{S}} n_{\mathrm{A}}-p_{\mathrm{A}}^{*} T_{2}^{\mathrm{S}}
\end{aligned}
$$

where $p_{\mathrm{A}}$ is the density of acceptors in the ground state. In steady state and assuming local neutrality, $n_{\mathrm{A}}=N_{\mathrm{D}}+p$, the model leads to an overall recombination rate $r(p)=$ $\left(1+\sigma F / T_{2}^{\mathrm{S}}\right) p\left(N_{\mathrm{D}}+p\right)$ which must be equated to the generation rate $g(p)=\sigma F\left(N_{\mathrm{A}}-N_{\mathrm{D}}-p\right)$, i.e. $r(p)=g(p)$, to calculate the free carrier concentration. The recombination rate increases superlinearly with rising free hole density $p$. This nonlinearity is of particular importance for low compensated materials because $p$ may become larger than $N_{\mathrm{D}}$ even for low intensities which negligibly depopulate the impurity ground state. This causes a saturation like intensity dependence of photoconductivity that in fact is due to nonlinear recombination rather than optical saturation. The experimental results indicate that for the intensity range of this investigation $p \ll N_{\mathrm{D}}$, in contrast to resonant photothermal ionization via metastable states. ${ }^{(14)}$ Therefore $r(p)$ may be linearized, $r(p) \approx\left(1+\sigma F / T_{2}^{\mathrm{S}}\right) p N_{\mathrm{D}}$. With this approximation the optically generated hole density $p(I)$ as function of intensity is given by

$$
p(I)=\left(N_{\mathrm{A}}-N_{\mathrm{D}}\right) \sigma\left(\frac{I}{\hbar \omega}\right) \tau_{\mathrm{r}}\left(1+\frac{I}{I_{\mathrm{S}}}\right)^{-1}
$$

in agreement to the experimental results. In this relation $\tau_{f}=\left(T_{1}^{\mathrm{S}} N_{\mathrm{D}}\right)^{-1}$ is the life time of holes in the band which in terms of the model is equal to the capture time in the excited statc. $I_{\mathrm{S}}=\hbar \omega / \sigma \tau_{\mathrm{r}}$ is the saturation intensity where $\tau_{\mathrm{r}}=\tau_{\mathrm{f}}+\tau_{2}$ is the total recombination time and $\tau_{2}=T_{2}^{\mathrm{s}-1}$ is the transition time between the excited state and the ground state.

The photoionization cross section $\sigma$ has been calculated using standard results of atomic physics and assuming the shallow acceptor to be hydrogen-like ${ }^{(17)}$. This gives $\sigma=7 \cdot 10^{-15} \mathrm{~cm}^{-2}$ for $\lambda=70.5 \mu \mathrm{m}$. The resulting recombination times $\tau_{\mathrm{r}}$ are listed in Table 1 . They are of the order of $10 \mu \mathrm{s}$ and vary with temperature and bias voltage. The capture time $\tau_{\mathrm{f}}$ may be determined from the decay of the photocurrent following a short laser pulse. Preliminary measurements applying a mode locked TEA- $\mathrm{CO}_{2}$ laser pumped $\mathrm{D}_{2} \mathrm{O}$ laser at $66 \mu \mathrm{m}$ wavelength yielded $\tau_{\mathrm{f}} \approx 50$ to $100 \mathrm{~ns}$ for $1 \mathrm{~V}$ bias voltage and $4.2 \mathrm{~K}$. Thus free holes are rapidly captured in excited acceptor states and need a much longer time to cascade across excited energy levels down to the acceptor ground state. The saturation intensity decreases and the recombination time increases with rising bias voltage. 
This behaviour must be attributed to excited state impact ionization which, due to the small binding energy of excited states, may be effective at voltages considerably lower than the threshold of instability. This interpretation explains also the dependence of the relative photoconductive signal $\Delta V / V$ on the bias voltage (Fig. 2). Impact ionization of excited states increases also the average capture time yielding a larger number of optically generated free carriers. The order of magnitude of the observed recombination times agrees quite well to that of Gershenzon et al ${ }^{(6)}$ extrapolated to the donor concentration of the present sample.

\section{CONCLUSION}

In summary the optical saturation of photoionization of shallow acceptors in very pure p-Ge has been investigated applying a high power FIR laser. From the intensity dependence of the photoconductive signal the saturation intensity and the recombination time of free holes was derived. The recombination time increases significantly with rising bias voltage indicating a slowing down of free carrier recombination due to impact ionization of acceptors in excited states. Complementary measurements of the decay of photoconductivity after irradiation by short laser pulses revealed that the capture time of free holes is about three orders of magnitude smaller than the recombination time.

Acknowledgements - We thank E E. Haller for provision of the sample. Financial support by the Deutsche Forschungsgemeinschaft is acknowledged.

\section{REFERENCES}

1. A Brandl, W. Kröninger, W. Prettl and G. Obermair, Phys. Ret Lett. 64, 212 (1990).

2. S. H. Koenig, R. D. Brown and W Schillinger, Phys, Rei, 128, 1688 (1962).

3. G. L. J. A. Rikken, P. Wyder, J. M. Chamberlain and L. L. Taylor, Europhys Lett. 5, 61 (1988).

4. J. Kaminski, J. Spector, W. Prettl and M. Welspfenning, Appl Phy's. Lett. 52, 233 (1988).

5. S. A. Kaufman, K. M. Kulikov and N. P. Likhtman, Sovtet Phys. Semicond. 4, 102 (1970)

6. E. M. Gershenzon, G. N. Goltsman, V. V. Multanovskii and N. G. Ptitsina, Soviet Phys. JETP 50, 728 (1979)

7. C. R. Pidgeon, A. Vass, G. R. Allan, W. Prettl and L. Eaves, Phys. Ret. Lett. 50, 1309 (1983).

8. G. R. Allan, A. Black, C. R. Pidgeon, E. Gornı, W Seidenbusch and P. Colter, Phys. Rev. B 31, 3560 (1985).

9. W. Prettl, A. Vass. G. R. Allan and C. R. Pidgeon, Int. J. infrared millimeter Waves 4, 561 (1983).

10. M. Weispfenning, I. Hoeser, W. Böhm, W. Prettl and E. Schöll, Phys, Ret. Lett. 55, 754 (1985).

11. Sh. M. Kogan and T. M. Lifshits, Physica status solidi (a) 39, 11 (1977)

12. M. Weispfenning, F. Zach and W. Prett!. Int. J infrared millimeter Waves 9, 1153 (1988).

13. G. Jungwirt, R. Kropf, U. Roessler and W. Prettl, Solid St. Commun. 72, 17 (1989).

14. G. Jungwirt, R. Kropf, W. Pretll and E. E. Haller. To be published

15. R. L. Jones and P. Fischer, J. Phys. Chem Solids 26, 1125 (1965).

16. M. Lax, Phys. Rev. 119, 1502 (1960).

17. H. A. Bethe and E. E. Salpeter, Quantum Mechanics of One and Two Electron Atoms. Springer. Berlın (1957) 